\title{
What's good for the goose, may not be good for the gosling
}

\author{
Jerrold Lerman MD FRCPC FANZCA
}

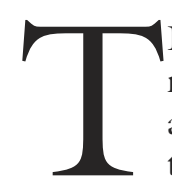

HE recent recall of nonprescription cough medicines in children has focused public attention on the "off-label"A use of medications in children (Washington Post, October 11, 2007). That the majority of drugs prescribed for children are "off-label" is well-established. Studies from a number of countries have consistently reported that up to $90 \%$ of drugs prescribed for infants and children are off-label, with some variability according to age and the location of care (i.e., outpatient $v s$ inpatient $p s$ intensive care unit). ${ }^{1-3}$ In the anesthesia literature, $66 \%$ of the studies published in 2003 in the journal Pain included off-label drugs and 34\% in the four leading anesthesia journals. ${ }^{4}$ In clinical pediatric anesthesia practice, the extent of "off label" drug use in children has not been quantified but is believed to be substantive. It seems appropriate then, to explore the polemic of off-label drug use in children from the perspective of the anesthesiologist.

It is purported that off-label drug use in children undergoing anesthesia is dangerous and unethical. The basis for these claims, in part, rests with the notion that the failure to develop a pediatric indication for these drugs has resulted in potentially beneficial off-label drugs being withheld while potentially harmful off-label drugs are prescribed. While there is arguably some truth to the notion (see below), ${ }^{5}$ pediatric anesthesiologists have assumed the burden of responsibility in this case investigating and publishing many studies to establish safe dosing guidelines for many off-label drugs that are now considered standard of care in infants and children. For example, the local anesthetic, bupivacaine, is not approved for epidural

\footnotetext{
A A term used to describe a drug that is not approved for either the indication for which it was prescribed/recommended and/ or for the age of the subject.
}

use in children less than $12 \mathrm{yr}$ of age but based on independent studies in neonates, infants and children, dosing regimens for both single-dose and continuous infusions are both widely and safely practiced today. ${ }^{6,7}$ Oral midazolam premedication for children was an off-label indication that was formulated independently by clinicians (by combining the parenteral drug with a vehicle) through a series of randomized controlled trials. Subsequently, the manufacturer developed a commercial preparation that has since been licensed for use in children. Some have even questioned the legality of prescribing off-label drugs to children. However, in two separate cases in the US, the decision by the court was that when the physician exercised his/her judgment to prescribe an off-label drug for a particular child, this practice constituted neither experimentation nor independent grounds for malpractice. ${ }^{8}$ Off-label drugs that are recognized as being unsafe in anesthesia have also been dealt with swiftly and decisively. Nineteen near-death experiences in children who received rapacuronium were reported to the Pediatric Anesthesia Conference discussion group in 2004. Within three months of publishing that data, ${ }^{9}$ rapacuronium was withdrawn from the market. These examples argue that high-quality evidence can and does exist beyond federally sanctioned approval and may be used to deliver safe and effective drugs as well as expunge those that may be dangerous from the market.

Notwithstanding our obligation to investigate and determine the suitability of off-label drugs for use in pediatric anesthesia, it is equally imperative that we reverse the practice of creating orphan drugs by developing an infrastructure to investigate and craft indications for both new and old drugs. In order to achieve this goal, we need to create an incentive for pharmaceuticals to pursue pediatric indications for

CAN J ANESTH 2008/55:2/pp 82-87

From the Department of Anesthesia, Women and Children's Hospital of Buffalo, SUNY at Buffalo, and Strong Memorial Hospital, University of Rochester, Rochester, New York, USA.

Address correspondence to: Dr. Jerrold Lerman, Department of Anesthesia, Women and Children's Hospital of Buffalo, 219 Byrant Street, Buffalo, New York 14222, USA. Phone: 716-878-7701; Fax: 716-878-7316; E-mail: jerrold.lerman@gmail.com

This work was not supported with external funding. Dr. Lerman holds neither conflicts of interest nor financial relationships with any company/product discussed in this manuscript. Reference is made to the off-label use of bupivacaine and propofol in children based on current federal approval for these products in Canada 
new drugs and develop a process by which current evidence is reviewed to determine whether an indication in children is warranted. The US has led the way to encourage pharmaceutical companies to pursue pediatric trials by extending drug exclusivity an additional six months when they complied. ${ }^{10,11}$ Furthermore, offlabel drugs in children have been prioritized and the current literature assessed to determine whether sufficient evidence already exists or to undertake additional studies to file indications for their use in children. Additionally, centres of excellence in pediatric research have been identified and funded to pursue trials with "off-label" as well as new drugs. As a result of these new strategies, between 1998 and 2002, the Food and Drug Administration (FDA) requested studies on 242 drugs and 53 were granted patent exclusivity. An additional 49 drugs received new labels. ${ }^{12}$ To date, no other country has emulated this level of commitment. In Canada as in the European Union, similar issues have been discussed, although neither funding nor concrete action has occurred.

The paucity of clinical trials in children has been attributed to a number of perceived obstacles to conducting research in this age group. ${ }^{11}$ In order to improve the quality of care that we deliver to children, we require high quality evidence, the foundation of which is the randomized controlled trial. The randomized control trial is designed to compare treatments when theoretical or clinical equipoise is present. When the purpose of the study is explained to parents in a clear and understandable manner and the risks to their child are minimal, the parents usually consent quite readily. However, many parents have a limited understanding of consent, randomization and other study design terminology. It is crucial that the team member who obtains consent from the parents makes the effort to explain the salient issues in an understandable manner, otherwise consent may not be granted. Even more difficult is approaching parents to obtain consent for a non-therapeutic study, which is a study in which the child may not directly benefit from participating (i.e., a pharmacokinetic study). Such was the case with a recent study on the pharmacology of dexmedetomidine in children. ${ }^{13}$ Not only did the children not substantively benefit from participating in the study, but blood was sampled from indwelling catheters for $24 \mathrm{hr}$ after the surgery. Because this was effectively a volunteer study, the consent rate was only $5 \%$. Finally, pharmaceutical companies are reluctant to sponsor indications in children because randomized controlled trials in children are expensive, recruiting children for studies is difficult, ethical and liability concerns abound and the pediatric market share is small, thus limiting the return on their investment.
The most controversial issue regarding off-label drug in pediatric anesthesia today has been reported apoptosis in the brains of neonatal rodents and small animals after exposure to commonly used anesthetics including ketamine, midazolam, isoflurane and nitrous oxide. ${ }^{14}$ Because the neonate is infrequently the subject of preclinical drug studies and because brain apoptosis has never been identified as a potential sequela associated with the use of these drugs, this serendipitous finding has raised widespread concern amongst scientists, anesthesiologists and parents alike. However, before these data are extrapolated to humans, there are many reasons why these observations may not be applicable to human neonates and children including differences in drug doses, rate of neurologic development and maturity and susceptibility to pharmaceutical insults among species. Indeed, in a recent investigation of perinatal Rhesus monkeys, there was no evidence of apoptosis subsequent to a three-hour infusion of ketamine. ${ }^{15}$ The final chapter on this subject remains to be written.

Propofol infusion syndrome (PRIS) is a life-threatening complication that usually presents in children (who are often septic) after $48 \mathrm{hr}$ of a propofol infusion at rates in excess of $5 \mathrm{mg} \cdot \mathrm{kg}^{-1} \cdot \mathrm{hr}^{-1} \cdot{ }^{15}$ A total of 61 patients have been diagnosed with PRIS, with 20 deaths in children and 18 in adults. ${ }^{16}$ Although Diprivan ${ }^{\circledR}$ has been approved for use in humans since 1989, PRIS was not reported until 1992. Propofol infusion syndrome appeared as a result of an off-label use of Diprivan ${ }^{\circledR}$ for long-term sedation in children. Concerns over the lethal nature of PRIS led the FDA to caution against the off-label use of propofol for sedation in children even though the evidence associating PRIS with brief sedation (less than four hours) in children has not been forthcoming. It would seem imprudent in 2008 to undertake any prolonged sedation with propofol in young children, particularly those with possible sepsis, but it would seem equally imprudent to proscribe the use of propofol for brief sedations for medical and surgical procedures (lasting less than four hours) without evidence. On the basis of these examples, it is clear that there is an urgent need for a mechanism to revise the package insert on a regular basis after a drug reaches the market thereby maintaining an up-to-date package insert.

The challenge that we face in 2008 and beyond is how to extricate ourselves from the plethora of off-label drugs and how to warrant a pediatric indication for new drugs in the future. No additional drugs should be approved for clinical use in children without an indication(s). A global strategy for drug approval should be developed in which redundant 
and overlapping studies from different countries are harmonized. By streamlining the drug development process, the reduced costs to develop new drugs will free up resources to conduct mandated pediatric trials. In concert with the US, other developed countries must institute programs to address the backlog of off-label drugs used in children. Finally, patient populations (i.e., neonates, infants and children) who are likely to use the new drug must be identified and large multi-country databases of safety and adverse events developed before drug approval. The post-marketing federal regulatory surveillance program for adverse drug reactions remains weak and underutilized. This process needs to be reinforced and physicians must be encouraged to report adverse events. Package inserts for all drugs must be updated regularly to reflect the current literature. It is only with a unified global approach that we can ensure that pediatric indications for new drugs are developed efficiently so that the highest quality care can be assured.

Whether or not you endorse the use of "off-label" drugs in pediatric anesthesia depends on your vantage point. From the child's point of view, "off-label" drugs may represent a new and better treatment. From the parent's point of view, "off-label" drugs may represent untested and unknown risks for which he/she has concerns and reticence. From the federal authority's point of view, "off-label" drugs represent serious concerns based in part, on unknown risks of and complications from the unregulated use of drugs over which the agency has no control. From the pharmaceutical executive's point of view, "off-label" drugs substantially raises annual sales and net profits, without investing in advertising and research or exposure from liability. From the anesthesiologist's point of view, "off-label" drugs may provide the standard of care or better with minimal risk based upon the best available evidence. In the final analysis however, your position on "off-label" drugs may be expressed best by Miles' Law: "Where you stand, depends on where you sit." 17

\section{Ce qui est bon pour l'oie ne l'est pas forcé- ment pour l'oison}

Le rappel récent de médicaments pour la toux sans ordonnance destiné aux enfants a attiré l'attention du public sur l'emploi «non conforme $»^{\mathrm{A}}$ (ou off-label) de médicaments chez les enfants (Washington Post, 11 octobre 2007). Que la plupart des médicaments prescrits chez les enfants le soient de façon «non conforme» est un fait avéré. Des études réalisées dans nombre de pays ont régulièrement observé que jusqu'à $90 \%$ des médicaments prescrits aux nourrissons et aux enfants le sont de façon non conforme, montrant une variation selon l'âge et le lieu de soins (c.-à-d. patient ambulatoire vs patient hospitalisé vs patient en soins intensifs). ${ }^{1-3}$ Dans la littérature anesthésique, $66 \%$ des études publiées en 2003 dans la revue Pain traitaient de médicaments utilisés de façon non conforme; ce chiffre s'élevait à $34 \%$ dans les quatre principales revues d'anesthésie. ${ }^{4}$ Dans la pratique clinique d'anesthésie pédiatrique, l'étendue de l'emploi non conforme de médicaments chez les enfants n'a pas encore été déterminée; toutefois, elle serait considérable. C'est pourquoi il convient d'explorer la polémique entourant l'emploi non conforme de médicaments chez les enfants du point de vue de l'anesthésiologiste.

On prétend que l'emploi non conforme de médicaments chez les enfants est à la fois dangereux et contraire à l'éthique. Ces affirmations s'appuient en partie sur l'idée que si une indication n'a pas pu être développée chez les enfants pour ces médicaments, cela a eu pour résultat que des médicaments non conformes mais potentiellement bénéfiques ne sont pas commercialisés alors que d'autres médicaments non conformes et potentiellement dangereux sont prescrits. Bien qu'il y ait sans doute une part de vérité dans cette conception de la chose (voir ci-dessous), ${ }^{5}$ les anesthésiologistes pédiatriques ont endossé la responsabilité de mener des recherches et de publier de nombreuses études dans le but d'établir des directives pour un dosage sécuritaire de plusieurs médicaments non conformes qui sont aujourd'hui considérés

A Terme utilisé pour décrire un médicament qui n'est pas approuvé soit pour l'indication pour lequel il a été prescrit/recommandé et/ou pour l'âge du patient. 
comme la norme des soins pour les nourrissons et les enfants. Par exemple, la bupivacaïne (l'anesthésique local) n'est pas approuvée pour un usage péridural chez les enfants de moins de douze ans. Néanmoins, sur la base d'études indépendantes menées chez des nouveaux-nés, des nourrissons et des enfants, les schémas posologiques pour des doses uniques aussi bien que des perfusions continues de bupivacaïne sont couramment appliqués à l'heure actuelle et ce, de manière tout à fait sécuritaire. ${ }^{6,7}$ La prémédication au midazolam par voie orale chez les enfants était une indication non conforme formulée de façon indépendante par des cliniciens (en combinant le médicament parentéral à un excipient) en se basant sur une série d'études randomisées contrôlées. Par la suite, le fabricant a conçu une préparation commerciale qui a depuis été brevetée pour être utilisée chez les enfants. Certains ont remis en question la légalité même d'une prescription de médicaments non conformes à des enfants. Cependant, dans deux cas différents aux États-Unis, la décision du tribunal a tranché que quand le médecin a exercé son propre jugement pour prescrire un médicament non conforme à un enfant en particulier, cette pratique n'était considérée ni comme de l'expérimentation, ni comme causes indépendantes de faute professionnelle. ${ }^{8}$ Quant aux médicaments non conformes dont on sait qu'ils ne sont pas sécuritaires en anesthésie, des mesures rapides et expéditives ont été prises. Dix-neuf expériences de mort imminente chez des enfants ayant reçu du rapacuronium ont été rapportées au groupe de discussion de la Conférence anesthésique pédiatrique en 2004. Trois mois après la publication de ces données, ${ }^{9}$ le rapacuronium a été retiré du marché. Ces exemples servent à démontrer que des données probantes de haute qualité peuvent exister - et existent - au-delà d'une approbation sanctionnée au niveau fédéral. Ces données peuvent être utilisées pour offrir des médicaments sécuritaires et efficaces ainsi qu'éliminer ceux qui pourraient être dangereux du marché.

Notre obligation d'examiner et de déterminer le caractère approprié de l'utilisation non conforme de médicaments en anesthésie pédiatrique est fondamentale; toutefois, il est tout aussi important de contrer la pratique actuelle de conception de médicaments orphelins en développant une infrastructure qui permette d'examiner et de forger des indications aussi bien pour les nouveaux médicaments que pour les anciens. Pour ce faire, il nous faut inciter les compagnies pharmaceutiques à approfondir les indications pédiatriques pour les nouveaux médicaments et développer un processus durant lequel les données probantes actuellement à disposition sont examinées afin de déterminer si une indication pédiatrique est de mise ou non. Les États-Unis ont été les premiers à encourager les compagnies pharmaceutiques à entreprendre des études en pédiatrie en prolongeant l'exclusivité du médicament de six mois supplémentaires si elles se plient à cette exigence. ${ }^{10,11}$ En outre, on a donné la priorité aux médicaments non conformes chez les enfants et évalué la littérature actuelle afin de déterminer s'il existe présentement suffisamment de données probantes ou s'il faut entreprendre des études supplémentaires pour enregistrer des indications pour leur usage en pédiatrie. De plus, des centres d'excellence en recherche pédiatrique ont été identifiés et subventionnés afin qu'ils entreprennent des études sur des médicaments non conformes ou nouveaux. Grâce à ces différentes stratégies, la Food and Drug Administration (FDA) américaine a exigé des études sur 242 médicaments, dont 53 ont reçu un brevet exclusif, et 49 ont reçu de nouvelles indications. ${ }^{12}$ À ce jour, aucun autre pays n'a démontré un tel niveau d'engagement. Au Canada et dans l'Union européenne, des questions similaires ont fait l'objet de discussions, mais aucune subvention ou action concrète n'a vu le jour.

Le manque d'études cliniques réalisées chez des enfants est traditionnellement attribué à plusieurs éléments considérés comme des obstacles à surmonter avant de pouvoir entreprendre des recherches dans cette catégorie d'âge. ${ }^{11}$ Toutefois, si nous voulons améliorer la qualité des soins que nous prodiguons aux enfants, nous avons besoin de données probantes de qualité élevée, et celles-ci se fondent sur des études randomisées contrôlées. L'étude randomisée contrôlée est conçue dans le but de comparer des traitements lorsque les données théoriques et cliniques s'équilibrent. Lorsque l'objectif de l'étude est expliqué aux parents d'une manière claire et compréhensible et que les risques encourus par leur enfant sont minimes, en général les parents acceptent volontiers sa participation. Cependant, nombre de parents n'ont qu'une compréhension limitée des termes employés, et des mots tels que 'consentement' ou 'randomisation' peuvent être mal interprétés. Il est donc crucial que le membre de l'équipe de recherche qui obtient le consentement des parents se donne la peine d'expliquer les questions fondamentales de manière compréhensible; sinon, le consentement pourrait être refusé. Il est encore plus difficile de demander le consentement aux parents pour une étude non thérapeutique, c'est-à-dire une étude qui pourrait ne pas procurer de bienfaits directs à l'enfant (par exemple, une étude pharmacocinétique). Tel a été le cas dans une étude récente sur la pharmacologie de la dexmédétomidine chez les enfants. ${ }^{13}$ Non seulement les enfants n'ont pas tiré de 
bienfaits réels de leur participation à l'étude, mais en plus des échantillons de sang ont été pris de cathéters à demeure pendant $24 \mathrm{~h}$ après l'opération. Étant donné qu'il s'agissait en réalité d'une étude menée sur une base volontaire, le taux de consentement n'a été que de $5 \%$. Enfin, les compagnies pharmaceutiques rechignent à subventionner des indications pour les enfants parce que les études randomisées contrôlées menées chez des enfants sont chères, le recrutement d'enfants est difficile, les questions éthiques et de responsabilité sont foison et la part du marché pédiatrique est limitée. Tout ceci limite le rendement du capital investi.

Actuellement, des cas d'apoptose observés dans les cerveaux de rongeurs nouveau-nés et de petits animaux après une exposition à des anesthésiques couramment utilisés comme la kétamine, le midazolam, l'isoflurane et protoxyde d'azote constituent la controverse la plus importante en ce qui touche à l'utilisation non conforme de médicaments chez les enfants. ${ }^{14}$ Étant donné que les rongeurs nouveauxnés font rarement l'objet d'études précliniques de médicaments et que l'apoptose du cerveau n'a jamais été identifiée comme séquelle potentielle associée à l'utilisation de ces agents, cette découverte fortuite a provoqué une inquiétude généralisée chez les scientifiques, les anesthésiologistes et les parents. Toutefois, avant d'extrapoler ces résultats à l'humain, il existe de nombreuses raisons pour lesquelles ces observations pourraient ne pas s'appliquer aux nouveau-nés et enfants humains, dont les différences de posologie, de niveau de développement neurologique, de maturité physiologique et de sensibilité aux agressions pharmaceutiques entre les espèces. D'ailleurs, dans une étude récente menée chez des macaques Rhesus périnataux, il n'y a pas eu d'apoptose après une perfusion de kétamine de trois heures. ${ }^{15}$ Cette question n'est donc pas encore résolue.

Le syndrome de perfusion du propofol (PRIS) est une complication extrêmement grave qui survient en général chez les enfants (qui sont souvent en septicémie) après $48 \mathrm{~h}$ de perfusion à plus de $5 \mathrm{mg} \cdot \mathrm{kg}^{-1} \cdot \mathrm{h}^{-1} \cdot{ }^{15}$ Au total, le PRIS a été diagnostiqué chez 61 patients, et il y a eu 20 décès chez des enfants et 18 chez des adultes. ${ }^{16}$ Bien que l'utilisation du Diprivan ${ }^{\circledR}$ chez des humains soit approuvée depuis 1989, aucun cas de PRIS n'a été rapporté avant 1992. Le syndrome de perfusion du propofol est apparu en raison d'une utilisation non conforme du Diprivan ${ }^{\circledR}$ pour une sédation prolongée chez des enfants. En raison d'inquiétudes quant à la nature fatale du PRIS, la FDA a émis un avertissement concernant l'utilisation non conforme du propofol pour sédationner des enfants et ce, même s'il n'y avait aucune donnée probante associant le PRIS à une sédation de courte durée (moins de quatre heures) chez les enfants. Il serait imprudent en 2008 d'utiliser le propofol pour induire quelque sédation prolongée que ce soit chez de jeunes enfants, et plus particulièrement ceux souffrant potentiellement de sepsis ; néanmoins, il serait tout aussi imprudent de proscrire l'utilisation de propofol pour des sédations brèves pour des procédures médicales et chirurgicales (durant moins de quatre heures) sans données à l'appui. En se fondant sur ces exemples, il est clair qu'il existe un urgent besoin pour un mécanisme qui permette de réviser la monographie du produit de façon régulière une fois que le médicament est sur le marché, de manière à garder la notice à jour.

Le défi majeur qui nous attend cette année et à l'avenir est de trouver un moyen de s'extirper de la surabondance de médicaments non conformes et de garantir une indication pédiatrique pour les nouveaux médicaments dans le futur. Aucun médicament supplémentaire ne devrait être approuvé pour être utilisé chez des enfants sans indication(s). Une stratégie mondiale devrait être élaborée pour l'approbation de nouveaux médicaments, dans laquelle les études de pays différents qui sont redondantes ou qui se recoupent sont harmonisées. En rationalisant le processus de développement du médicament, les coûts ainsi réduits libéreront des ressources pour mener des études pédiatriques commanditées. D'autres pays développés doivent, de concert avec les États-Unis, établir des programmes afin de se charger des médicaments non conformes utilisés chez les enfants qui n'ont pas encore fait l'objet d'études rigoureuses. Enfin, les populations à l'étude (c.-à-d. les nouveau-nés, les nourrissons et les enfants) qui pourraient utiliser le nouveau médicament doivent être identifiées et des bases de données conséquentes, recoupant plusieurs pays, portant sur l'innocuité et les effets secondaires doivent être mises en place avant l'approbation d'un nouveau médicament. Le programme fédéral réglementaire de pharmacovigilance pour les effets indésirables demeure faible et n'est pas pleinement utilisé. Ce programme doit être renforcé et les médecins doivent être encouragés à rapporter les effets indésirables. Les monographies de tous les médicaments doivent être régulièrement mises à jour afin de refléter les données actuelles présentées dans la littérature. Seule une approche mondiale unifiée nous permettra d'assurer que les indications pédiatriques pour de nouveaux médicaments sont développées de façon efficace, de manière à assurer une meilleure qualité de soins.

Le fait d'approuver ou non l'utilisation de médicaments non conformes en anesthésie pédiatrique 
dépend du point de vue. Du point de vue de l'enfant, les médicaments non conformes pourraient représenter un traitement nouveau et plus efficace. Du point de vue des parents, les médicaments non conformes pourraient constituer des risques non testés et inconnus à cause desquels ils sont inquiets et réticents. Du point de vue des autorités fédérales, les médicaments non conformes représentent de sérieuses inquiétudes, en partie à cause des risques inconnus et des complications associés à l'utilisation non réglementée de médicaments sur laquelle l'agence n'a aucun contrôle. Du point de vue du dirigeant de compagnie pharmaceutique, les médicaments non conformes augmentent considérablement les ventes annuelles et le bénéfice net, sans nécessiter d'investissement dans la publicité et la recherche et n'exposant pas la compagnie à une quelconque responsabilité. Du point de vue de l'anesthésiologiste, les médicaments non conformes pourraient offrir une norme de soins ou même mieux avec un minimum de risques sur la base des meilleures données probantes à disposition. Au bout du compte, votre opinion sur l'utilisation des médicaments non conformes peut être résumée par la loi de Miles : «Le point de vue dépend d'où l'on se place. ${ }^{17}$

\section{References}

1 Shah SS, Hall M, Goodman DM, et al. Off-label drug use in hospitalized children. Arch Pediatr Adolesc Med 2007; 161: 282-90.

2 Pandolfini $C$, Bonati $M$. A literature review on off-label drug use in children. Eur J Pediatr 2005; 164: 552-8.

3 Conroy S, McIntyre J, Choonara I. Unlicensed and off label drug use in neonates. Arch Dis Child Fetal Neonatal Ed 1999; 80: F142-5.

4 Kopacz DJ. The use of "off-label" medications in anesthesia journal publications. Anesthesiology 2004; 101: A-1310.

5 Horen B, Montastruc JL, Lapeyre-Mestre M. Adverse drug reactions and off-label drug use in paediatric outpatients. Br J Clin Pharmacol 2002; 54: 665-70.

6 Gunter JB, Dunn CM, Bennie JB, Pentecost DL, Bower $R J$, Ternberg JL. Optimum concentration of bupivacaine for combined caudal - general anesthesia in children. Anesthesiology 1991; 75: 57-61.

7 Berde CB. Convulsions associated with pediatric regional anesthesia. Anesth Analg 1992; 75: 164-6.

8 Meblman MJ. Off-label prescribing. May 2005. Available from URL; www.thedoctorwillseeyounow. com/articles/bioethics/offlabel_11/(accessed November 16, 2007).

9 Meakin GH, Pronske EH, Lerman J, et al. Bronchospasm after rapacuronium in infants and children. Anesthesiology 2001; 94: 926-7.
10 Steinbrook $R$. Testing medications in children. New Engl J Med 2002; 347: 1462-70.

11 Caldwell PH, Murphy SB, Butow PN, Craig JC. Clinical trials in children. Lancet 2004: 364; 803-11.

12 Roberts R, Rodriguez W, Murphy D, Crescenzi T. Pediatric drug labeling: improving the safety and efficacy of pediatric therapies. JAMA 2003; 290: 905-11.

13 Petroz GC, Sikich N, James M, et al. A phase 1, two-center study of the pharmacokinetics and pharmacodynamics of dexmedetomidine in children. Anesthesiology 2006; 105: 1098-110.

14 Mellon RD, Simone AF, Rappaport BA. Use of anesthetic agents in neonates and young children. Anesth Analg 2007; 104: 509-20.

15 Slikker W Jr, Zou X, Hotchkiss CE, et al. Ketamineinduced neuronal cell death in the perinatal Rhesus monkey. Toxocol Sci 2007; 98: 145-58.

$16 \mathrm{Kam} \mathrm{PC,} \mathrm{Cardone} \mathrm{D.} \mathrm{Propofol} \mathrm{infusion} \mathrm{syndrome.}$ Anaesthesia 2007; 62: 690-701.

17 Miles RE Jr. The origin and meaning of Miles' Law. Public Adm Rev 1978; 38: 399-403. 